\title{
A CASE OF KLINEFELTER'S SYNDROME WITH HIGH INTELLIGENCE LEVEL
}

\author{
Shinichiro NANKo* \\ Institute of Brain Research, School of Medicine, University of Tokyo, \\ Bunkyo-ku, Tokyo 113, Japan
}

\begin{abstract}
Summary A male of Klinefelter's syndrome with I.Q. more than 140 by WAIS, found among university students was reported. Recent surveys among general population showed that intelligence level of this syndrome was around normal level. These results indicate that the impaired intellectual is not necessary for Klinefelter's syndrome.
\end{abstract}

\section{INTRODUCTION}

Males with Klinefelter's syndrome has been believed to be associated with impaired intellectual level. This is based on surveys among special population, for example those mentally retarded or mentally ill. Recent surveys among general populations, such as newborns showed that intelligence level of Klinefelter's syndrome was around normal level (Ratcliffe et al., 1979, 6 cases, mean I.Q. 104.3; Nielsen et al., 1979, 4 cases, mean I.Q. 103.3). Barker and Black (1976) surveyed a military population and found nine patients with this syndrome. Their mean I.Q. was 104.5. We surveyed this syndrome among university students and found one male with high intelligence level.

\section{CASE FINDING PROCEDURE}

The subjects were 1,280 male and 981 female university students aged 18-24 years. With the consent of the subjects, $\mathrm{X}$ chromatin screening by cresyl-violet staining of buccal smear was performed in both sexes, and $Y$ chromatin by quinacrine mustard staining of buccal smear only in males. Among 1,280 males, a male with positive $X$ chromatin was found $(0.08 \%)$. Peripheral lymphocyte culture of this male showed 47,XXY karyotype. No numerical abnormalities of X and Y chromatin were found except this case.

Received February 2, 1983

* Present address: Department of Psychiatry, School of Medicine, University of Teikyo, Jtabashiku, Tokyo 173, Japan 


\section{Case}

M.I. 24 years of age, $184 \mathrm{~cm}$ in height, $48 \mathrm{~kg}$ of weight. The father was 37 years old and the mother 27 at the time of his birth. He has a younger sister. There was no history of psychiatric-psychological and/or physical problem except suicide attempt when he failed entrance examination to high school at the age of 15. Physical examinations were compatible with Klinefelter's syndrome, which were scanty axillary and pubic hair, small testis, elastic skin surface and gynecomastia. Psychological testings showed that he was passive but imaginable person and adapted well to his circumstances. WAIS showed that Full Scale I.Q. was more than 140, which was a maximum score of this test. Verbal I.Q. was more than 140 and performance I.Q. 134 .

\section{COMMENT}

This is a highest I.Q. level of the males with Klinefelter's syndrome ever reported, following is a case with I.Q. 134 reported by Puck et al. (1975).

Although the population examined is small, the frequency of XXY male among university male students, who are suspected to have a higher intelligence level than the general population, in this survey $(0.08 \%)$ is quite the same figure compared to those of newborns $(0.09 \%$, Hook and Hamerton, 1977).

These results and recent surveys among general population reported indicate that the impaired intellectual level is not necessary for Klinefelter's syndrome. This might be encouraging to parents who have a child with this syndrome, as well as the cytogenetists who are conducting the newborn survey and counselling of parents.

\section{REFERENCES}

Barker, T.E., and Black, F.W. 1976. Klinefelter syndrome in a military population. Arch. Gen. Psychiat. 33: 607-610

Hook, E.B., and Hamerton, J.L. 1977. The frequency of chromosome abnormalities detected in consecutive newborn studies. In Population Cytogenetics: Studies in Human's (Hook, E.B. and Porter, I.H., eds.), Academic Press, New York

Nielsen, J., Sillesen, I., Sørensen, A.M., and Sørensen, K. 1979. Follow-up until age 4 to 8 of 25 unselected children with sex chromosome abnormalities, compared with sibs and controls. In Sex Chromosome Aneuploidy: Prospective Studies on Children (Robinson, A., Lubs, H.A., and Bergsma, D., eds.), pp. 15-73, A.R. Liss Inc., New York

Puck, M., Tennes, K., Frankenburg, W., Bryant, K., and Robinson, A. 1975. Early childhood development of four boys with 47,XXY karyotype. Clin. Genet. 7: 8-20

Ratcliffe, S.G., Axworthy, D., and Ginsborg, A. 1979. The Edinburgh study of growth and development in children with sex chromosome abnormalities. In Sex Chromosome Aneuploidy: Prospective Studies on Children (Robinson, A., Lub., H.A., and Bergsma, D., eds.), pp. 243-260, A.R. Liss Inc., New York 